\title{
La question du rapport au corps dans la formation en STAPS : Cas de deux enseignants universitaires tunisiens
}

\author{
Salma Majdoub Chtara, PhD candidate \\ École doctorale : DISEMEF, UR ECOTIDI (UR16ES10) \\ ISEFC, Université virtuelle de Tunis
}

\section{Hejer Ben Jomâa, PhD}

Institut Supérieur du Sport et de l'Education Physique de Ksar-Saïd,

Université de La Manouba, Tunisie

UR ECOTIDI (UR16ES10), ISEFC, Université virtuelle de Tunis Unité Mixte de Recherche (UMR) sur l'Education, la Formation, le Travail et le Savoir (EFTS), Université Toulouse Jean-Jaurès

\section{Hiba Abdelkafi Karoui, PhD candidate}

École doctorale : DISEMEF, UR ECOTIDI (UR16ES10), ISEFC

Université virtuelle de Tunis

Abdelmadjid Naceur, PhD

UR ECOTIDI (UR16ES10), ISEFC, Université virtuelle de Tunis

\section{Georges Kpazaï, PhD}

Groupe de Recherche sur l'Évaluation et le Développement des

Compétences en Activité Physique et en Santé (GRÉDCAPS)

École des sciences de l'activité physique (ÉSAP), Faculté de la santé,

Université Laurentienne, Sudbury (Ontario), Canada

Doi:10.19044/esj.2020.v16n10p262 URL:http://dx.doi.org/10.19044/esj.2020.v16n10p262

\section{Résumé}

Comment le corps de l'enseignant universitaire expérimenté se déploie-t-il en formation pratique STAPS ? Cette étude comparative de l'effet du rapport au corps de deux enseignants universitaires expérimentés sur leurs pratiques enseignantes se base sur une double filiation théorique : didactique et clinique d'inspiration psychanalytique (Terrisse \& Carnus, 2009 ; Carnus \& Terrisse, 2013). Elle constitue le prolongement des travaux de l'EDiC sur la didactique universitaire (Sghaier, Ben Jomâa, Mami, Kpazaï, \& Bouassida, 2019 ; Ben Jomâa, Abdelkefi, Chili, Majdoub, \& Kpazaï, 2018) et se réfère ainsi au cadre théorique de la didactique clinique universitaire à perspective de formation (Romainville, 2002 ; Altet, 1994 ; Perrenoud, 1994). Le primat accordé à l'analyse de la place du rapport «différentiel» au corps dans 
l'enseignement supérieur, à travers le prisme de l'expérience professionnelle et au regard du genre (Ben Jomâa, 2019) vise à étudier les articulations entre rapport au corps, genre et action didactique. Cette prise en compte a priori et a posteriori de la pratique enseignante s'organise à travers deux études de cas rapprochés, dont nous avons l'intention d'examiner et d'en comparer notamment le rapport au corps (féminin vs masculin) et son rôle dans les choix didactiques, quant à l'usage de la distance «didactique », des formes ostensives et des formes de proxémie sous un angle clinique. Cette recherche se veut descriptive et compréhensive. Elle se focalise sur l'analyse des ressorts de l'acte d'enseignement au supérieur à travers une épistémologie pratique privée, un déjà-là singulier et des postures professorales différenciées. Les résultats de la présente recherche ont permis d'identifier un rapport « différentiel au corps » qui se déploie. L'enseignant masculin a un rapport techniciste au corps, tandis que l'enseignante féminin a un rapport régulateur au corps. Ce rapport «différentiel au corps » est révélateur d'un rapport au savoir singulier, propre à l'histoire de chaque enseignant.

Mots clés : Didactique universitaire, pratique enseignante, rapport au corps, ostension, étude de cas 


\title{
The question of the link to body in STAPS initial training: The case of two Tunisian university teachers
}

\author{
Salma Majdoub Chtara, PhD candidate
}

École doctorale : DISEMEF, UR ECOTIDI (UR16ES10)

ISEFC, Université virtuelle de Tunis

\section{Hejer Ben Jomâa, PhD}

Institut Supérieur du Sport et de l'Education Physique de Ksar-Saïd,

Université de La Manouba, Tunisie

UR ECOTIDI (UR16ES10), ISEFC, Université virtuelle de Tunis

Unité Mixte de Recherche (UMR) sur l'Education, la Formation, le Travail et le Savoir (EFTS), Université Toulouse Jean-Jaurès

\section{Hiba Abdelkafi Karoui, PhD candidate}

École doctorale : DISEMEF, UR ECOTIDI (UR16ES10), ISEFC

Université virtuelle de Tunis

Abdelmadjid Naceur, PhD

UR ECOTIDI (UR16ES10), ISEFC, Université virtuelle de Tunis

\section{Georges Kpazaï, PhD}

Groupe de Recherche sur l'Évaluation et le Développement des

Compétences en Activité Physique et en Santé (GRÉDCAPS)

École des sciences de l'activité physique (ÉSAP), Faculté de la santé,

Université Laurentienne, Sudbury (Ontario), Canada

\begin{abstract}
How can the university teacher's body be deployed in the STAPS initial training? This comparative study aims to examine the effect of the link to body of two experimented university teachers on their teaching practice. It is based on a double theoretical filiation: didactical and clinical from a psychoanalytical inspiration (Terrisse \& Carnus, 2009 ; Carnus \& Terrisse, 2013). This study follows the EDiC works on the higher education didactics (Sghaier, Ben Jomâa, Mami, Kpazaï, \& Bouassida, 2019; Ben Jomâa, Abdelkefi, Chili, Majdoub, \& Kpazaï, 2018) and refers to its conceptual framework from a training perspective (Romainville, 2002 ; Altet, 1994 ; Perrenoud, 1994). A primacy is granted to the analysis of the 'differential' link to body of the teacher through the professional experience and the gender (Ben Jomâa, 2019). This aims to study the linkage between the link to body, gender, and the didactics action. We take into account the teaching practice a priori
\end{abstract}


and a posteriori through two reconciled cases-study which we intend to examine and to compare their link to body (feminine vs masculine). This comparison plan to examine the role of the didactic choices regarding the use of didactic distance, ostensive and proxemics forms. This study is mainly descriptive and comprehensive. It focuses on the analysis of the motives of the teaching act in higher education through a private practical epistemology, a singular 'already-there' and differentiated professorial postures. The results of this research have enabled us to identify a "differential to the body" relationship that deploys. The male teacher has a technical relationship to the body, while the female teacher has a regulatory relationship to the body. This "differential to the body" report is indicative of a relationship with singular knowledge, specific to the history of each teacher.

Keywords: Higher education didactics, teaching practice, link to body, ostension, case-study

\section{Introduction}

Cette étude se situe dans le prolongement d'une réflexion autour d'une thèse de doctorat en cours d'élaboration ${ }^{5}$. Elle s'inscrit dans l'orientation scientifique de la didactique clinique (Terrisse \& Carnus, op. Cit. ; Carnus \& Terrisse, op. Cit) et dans l'ouverture récente des travaux de $1^{\prime} \mathrm{EDiC}^{6}$ sur la didactique universitaire (Ben Jomâa et al., 2018 ; Ben Jomâa, 2019 ; Sghaier et al., 2019). Elle articule ainsi « recherche et formation » en faisant appel à la formation pratique en $\mathrm{STAPS}^{7}$, telle qu'elle se déploie aux Instituts Supérieurs du Sport et de 1'Education Physique (ISSEP) en Tunisie ${ }^{8}$. Cette recherche pointe la problématique du rapport au corps de l'enseignant universitaire expérimenté et spécialiste d'une discipline sportive particulière ${ }^{9}$, face à l'insuffisance du cadre d'analyse verbal dans la compréhension et l'analyse des pratiques enseignantes universitaires in situ.

Pour ce faire, nous examinons l'effet des stratégies ostensives (Salin, 2002) et proxémiques (Hall, 1966-1971 ; Forest, 2006) sur la transmission du savoir. Pujade-Renaud (1984) estime que la proxémie permet d'avoir la maîtrise du jeu de rapprochement et de distanciation par rapport aux élèves,

\footnotetext{
${ }^{5}$ Thèse de doctorat en cours d'élaboration dans le cadre structuré de l'Ecole Doctorale DISEMEF, ISEFC, Université virtuelle de Tunis, Tunisie.

${ }^{6}$ Equipe de Didactique Clinique, Unité Mixte de Recherche (UMR) sur l'Education, la Formation, le Travail et le Savoir (EFTS), à l'université de Toulouse Jean-Jaurès en France (UT2).

${ }^{7}$ Sciences et Techniques des Activités Physiques et Sportives.

${ }^{8}$ Formation universitaire en Sciences et Techniques des Activités Physiques et Sportives dans le cadre des instituts supérieurs du sport et de l'éducation physique (ISSEP) en Tunisie, le cas de l'ISSEP de Ksar-Saïd (Tunis).

${ }^{9}$ Discipline enseignée : L'athlétisme.
} 
mais aussi par rapport aux savoirs (Ben Jomaa et al., 2018). Cette « verbalisation du corps » représente « l'ensemble des comportements et des processus psychologiques qui permettent de transmettre et de recevoir des informations »(Clément \& Noels, 2006). Ceci fait penser aux dimensions corporelles de la pratique enseignante à travers les gestes d'études (ostension, proxémie, distance et posture) utilisés en situation interactive et contingente de classe.

Nous tenterons de répondre aux questions de recherche suivantes : comment se déploie le corps de l'enseignant universitaire spécialiste d'une activité physique et sportive (APS) donnée lors d'une situation de transmission de savoirs? Comment «verbalise »-t-il son corps lors de l'épreuve d'enseignement et quels en sont les enjeux du savoir (Ben Jomaa, 2009)? Quelle posture corporelle, épistémologique et professionnelle adopte-t-il selon que son corps est masculin ou féminin? En quoi et comment son rapport au corps « différentiel » organise-t-il sa pratique enseignante?

\section{Cadre conceptual}

Malgré les études multiples sur le corps de l'enseignant d'EPS, la place du corps de l'enseignant d'EPS dans sa classe a peu été investiguée (Boizumault \& Cogérino, 2012). En 1971, Mehrabian évoque que le registre de la corporéité reste «implicite et difficilement verbalisable ». Un regard clinique sur le sujet permet d'en gagner en compréhension. Dans l'exercice de leur profession, les enseignants universitaires sont confrontés systématiquement à des défis particulièrement importants. Ils sont interrogés dans leurs décisions professionnelles qui sont déterminées par leurs histoires intimes et leurs rapports aux savoirs disciplinaires, voir même professionnels. Il s'agit de rompre avec l'idée d'un sujet générique et épistémique et l'inscrire dans une théorie du sujet pris dans le didactique en relation avec le savoir en jeu, l'épreuve de l'interaction, marquée par la contingence du processus enseignement-apprentissage et assujettie par l'institution au sein de laquelle se déroule le processus de transmission-appropriation de savoirs (Carnus \& Terrisse, 2013 ; Terrisse \& Carnus, 2009).

Cette théorie de sujet en didactique clinique (Terrisse \& Carnus, 2009) modélisée à partir d'un triangle didactique revisité trouvant ses origines dans l'approche systémique ternaire de Sensevy (2007), considère le sujet enseignant comme étant singulier, assujetti et divisé. Des mécanismes « conscients » mais aussi « inconscients ${ }^{10}$ influencent de manière latente et

${ }^{10}$ Nous nous référons à l' « hypothèse freudienne » (Sauret, 2000), qui intègre de manière explicite la notion de l'inconscient, source de nombreuses divisions chez le sujet enseignant, et qui permet, entre autres, d'éclairer une part d'insu dont nous avons pu faire à maintes reprises le constat, notamment dans le remaniement des contenus d'enseignement (Carnus, 2009 ; Ben Jomâa, 2009). 
constante les choix didactiques du sujet enseignant et orientent ainsi, souvent à son insu, certaines de ses actions didactiques, ses conceptions, ses intentions ainsi que ses décisions didactiques.

Tous ces filtres personnels déterminent le déjà-là décisionnel ${ }^{11}$ (Carnus, 2001, 2003, 2015) et révèlent la complexité et la multiplicité des influences internes possibles lors de la prise de décision au moment de l'épreuve interactive (Carnus \& Terrisse, 2013, p.142). Lameul (2008) précise que la posture que nous adoptons en tant qu'enseignant est singulière et individualisée du fait qu'elle est « façonnée par nos croyances et orientée par nos intentions. Elle exerce une influence directrice et dynamique sur nos actions, leur donnant sens et justification ». Ce corps «parlant» de l'enseignant permet aussi de communiquer un savoir et favorise «l'enrôlement des étudiants dans les tâches didactiques » (Jorro, 2006). En effet, en tant qu'intervenant, son corps est assujetti constamment, au regard des apprenants qui s'intéressent parfois plus à « son corps qu'à son langage » (Maulini \& Olivier, 1999). Pour transmettre le savoir, il doit apprendre à mettre son corps en scène et à le faire parler.

Il s'agit d'un investissement corporel s'inspirant d'un « déjà-là expérientiel sportif » (Loizon, 2016). Ce «savoir y faire » (Terrisse, 2001) tend à se convertir en contenus d'enseignement et/ou en savoirs expérientiels (Buznic-Bourgeacq, 2009). Investir le champ de la régulation didactique présente un défi relationnel et quotidien pour chaque enseignant. Ce mode de fonctionnement didactique différent, souvent utilisé «pour montrer, démontrer, corriger à des distances variées » (Magali, \& Geneviève, 2012), s'apparente à celui qui joue un jeu de rôle et qui construit une relation d'aide. De plus, la régulation est un geste qui demande des déplacements dans des espaces relativement étendus et des compétences évaluatives (Durand, 2001 ; Cauvin, 2002 ; Buznic-Bourgeacq, Terrisse \& Margnes, 2007 ; Carnus, 2008) en vue de rendre l'action didactique plus efficace.

Ce ressenti corporel est caractérisé par « des mécanismes de régulation, de guidage, de contrôle, d'ajustement et de réorientation de l'action » (Allal \& Mottier-Lopez, 2007). La régulation de l'action est comprise comme «un ajustement des dispositifs pédagogiques ou enseignants, par le biais d'opérations précises, en fonction des besoins des étudiants » (MottierLapez, 2013). En effet, ces pratiques de régulation reviennent ainsi pour nous à connaître comment les enseignants s'y prennent pour favoriser les apprentissages des étudiants, et par-là, de mettre à jour des phénomènes en lien avec la façon dont est « géré » et « mis en scène » le savoir (Amade Escot,

${ }^{11}$ Une notion qui porte en elle différents aspects du rapport au(x) savoir(s) : Structurels au regard de ses éléments constitutifs conceptuel, intentionnel et expérientiel ; fonctionnels au vu de ses influences sur les savoirs enseignés et/ou appris ; dynamiques compte tenu de son caractère forcément évolutif au sein d'une temporalité construite (Carnus, 2015, p.64) 
2002). L'enseignant au moment de sa pratique enseignante utilise des objets ou des représentations symboliques (Buznic-Bourgeacq, Terrisse \& Lestel, 2008 ) et des manipulations corporelles pour favoriser un mouvement ou une construction de sensations. Ce corps régulateur peut alors « donner à voir »du savoir au travers de procédures ostensives (Salin, 2002).

Cette intervention corporelle régulatrice de l'enseignant universitaire, intervenant dans le cadre de la formation pratique en STAPS, est sensible à la problématique de la régulation en termes de «technicité » et de « recherche d'efficacité » souvent technique (Joas, 2001). Loizon, Margnes et Terrisse (2005) définissent la technique comme étant «la manifestation la plus directement observable, essentiellement gestuelle et spatiale ». D'après ces auteurs (Loizon et al., Op. Cit), la technique conduit à l'efficacité gestuelle. Le corps de l'enseignant techniciste (Marsenach \& Mérand, 1987 ; AmadeEscot, 1991) pourrait s'avérer efficace dans l'enseignement (Lafont, 1994 ; Kohler, 2002) et donnerait à l'étudiant un rôle actif dans son apprentissage.

Dans le cadre de cet article, nous faisons le choix de mener une analyse fine de la pratique enseignante universitaire à travers trois analyseurs didactiques et/ou cliniques : 1) le rapport au savoir, 2) le rapport au corps et 3) "l'impossible à supporter » (Lacan, 1967). Ce dernier analyseur clinique, qui appartient à l'univers psychanalytique, manifeste une pertinence dans l'observation des pratiques d'enseignement (Touboul, 2013). Il a été défini d'après Lacan (1977) comme étant « le réel en tant qu'il est l'impossible à supporter ». C'est le réel indécidable surtout au moment de l'épreuve puisque cette dernière est imprévisible, contingente et incertaine. L'enseignant se trouve obligé de faire des régulations et de prendre de nouvelles décisions. Son action éducative influence ses intentions et ses choix didactiques (Loizon, 2009). Il s'agit de sa partie non-visible, donc cachée, de son processus décisionnel.

L'enseignant qu'il soit homme ou femme, pouvait s'engager autant par sa propre «épistémologie pratique » (Sensevy, 2007), ses affects et ses intentions en classe. Cet article souligne une prééminence qui serait accordée au corps de l'enseignant en tant que médiateur des savoirs, porteur d'une histoire, d'un rapport au corps, d'un rapport à l'institution et d'un rapport au savoir disciplinaire. A cet égard, l'enseignant mobilise un registre corporel identitaire et irréductiblement singulier dans un espace didactique théâtralisé, « un champ de bataille, un lieu à investir et à maitriser » (Pujade-Renaud, 1984).

\section{Cadre méthodologique}

D'un point de vue méthodologique, l'étude se fait au cas par cas (Terrisse, 2003). Cet outil méthodologique clinique s'attache à décrire les pratiques enseignantes ordinaires non prescriptives. L'étude de cas prend en 
considération une théorie du sujet singulier, divisé, assujetti et pris dans le didactique. En effet, l'usage de cette dernière amène les chercheurs de l'EDiC à rendre compte de la singularité d'un sujet enseignant à partir de l'analyse de ses choix didactiques et de son comportement en classe. Ce qui permet également de poursuivre la quête d'une intelligibilité des pratiques observées. Dans ce contexte, nous retenons le point de vue de Passeron et Revel (2005) qui estiment que « les sciences du cas ont ainsi assuré la convergence entre la valeur descriptive de la méthode clinique et la valeur méthodologique de l'observation contextualisée dans l'administration de la preuve ».

Dans le cadre de cet article, nos choix méthodologiques portent sur l'analyse de deux cas. Nous optons pour une étude de cas rapprochés. Il s'agit d'observer et d'analyser la pratique enseignante de deux enseignants universitaires expérimentés et spécialistes d'athlétisme : une femme et un homme ayant le grade de maître-assistant universitaire. Le tableau suivant décrit le profil des deux enseignants associés à la présente recherche : cas Samar ${ }^{12}$ et cas $\mathrm{Ali}^{13}$ (tableau 1).

Tableau 1 : Statut professionnel des deux enseignants participants à la recherche.

\begin{tabular}{lcc}
\hline $\begin{array}{l}\text { Enseignant (e)s } \\
\text { universitaires }\end{array}$ & Cas Samar & Cas Ali \\
\hline Grade & Maître-assistante & Maître-assistant \\
\hline Expérience professionnelle & 18 ans & +18 ans \\
\hline Expérience pratique & 10 ans & +10 ans \\
\hline
\end{tabular}

\section{Stratégies de recueil des données}

Nous avons observé les pratiques effectives de Samar et Ali durant quatre cours d'athlétisme. Chacun des deux enseignants participant à la recherche s'est engagé pour l'enseignement de deux cours. La durée de ces cours pratiques a été limitée par l'institution à une heure et demie. Le recueil des données s'est organisé ainsi autour de l'observation in situ ${ }^{14}$ et de la

\footnotetext{
${ }^{12}$ Samar : Maître-assistante universitaire spécialiste d'athlétisme.

${ }^{13}$ Ali : Maître-assistante universitaire spécialiste d'athlétisme.

${ }^{14}$ L'observation in situ se présente comme une méthode de recherche qui intercepte «les comportements au moment où ils se produisent sans l'intermédiaire d'un document ou d'un témoignage » (Quivy \& Van Campenhoudt, 2006). Cette observation ethnographique systématique non participante et directe a été assurée par deux caméras et un micro-cravate pour avoir des enregistrements audio et vidéo.
} 
pratique des entretiens ante ${ }^{15}$ et post séance ${ }^{16}$. Notre corpus s'est constitué en fait d'enregistrements audio (du verbatim des enseignants) et vidéo de quatre cours d'athlétisme. L'objectif de cette phase de recueil de données demeure l'accès au comportement du sujet enseignant, aux raisons de ses actes ainsi qu’à sa réalité psychique.

\section{Stratégies d'analyse des données}

Nous notons que ce type de recherche qualitative repose sur un ensemble de techniques d'analyse de données dont l'usage est très répandu. Dans le cadre de ce travail, nous avons fait recours à la technique d'analyse de contenu (Bardin, 1998) et à celle de triangulation méthodologique des données (Van Der Maren, 1996 ; Savoie-Zajq, 1996) selon un mode de validation interne de recherche. Pour ce faire, nous avons procédé en premier temps par une lecture flottante puis détaillée (Bardin, 1998) des données recueillies. En second temps, nous avons repéré les faits remarquables et les événements significatifs à l'égard de notre problématique de recherche. En troisième temps, nous avons découpé les unités de sens (ou significatives) grâce à la technique du codage. Finalement, nous avons classé ces unités de sens selon des catégories thématiques permettant, une fois analysées, d'interpréter les données.

Dans ce contexte, nous nous référons à la méthodologie didactique clinique (Terrisse \& Carnus, 2009) telle qu'elle a été élaborée, décrite et utilisée par les chercheurs de l'EDiC. Cette méthodologie temporelle s'organise ainsi autour de trois temps distincts mais complémentaires (tableau 2) : le déjà-là, l'épreuve et l'après-coup (Terrisse \& Carnus, 2009).

Tableau 2 : Temporalité de la méthodologie didactique clinique

(Terrisse, Carnus \& Sauvegrain, 2002)

\begin{tabular}{|l|c|c|}
\hline $\begin{array}{l}\text { Déjà-là (Carnus, } \\
\text { 2003) }\end{array}$ & $\begin{array}{c}\text { Filtre de l'action didactique } \\
\text { (Loison, 2009, 2013) } \\
\text { Epreuve }\end{array}$ & Planification \\
\hline Après-coup & $\begin{array}{c}\text { Meconseignante in situ } \\
\text { Recoution et réorganisation des } \\
\text { événements }\end{array}$ & Remaniement du sens \\
\hline
\end{tabular}

${ }^{15}$ L'entretien ante séance est un entretien semi-dirigé qui s'organise dans les minutes qui précèdent la phase de l'interaction didactique et permet de recueillir des données relatives aux planifications des séances et/ou du "savoir à enseigner", qui seront en mesure d'orienter le chercheur dans l'analyse du déjà-là intentionnel et conceptuel du sujet enseignant.

${ }^{16}$ L'entretien post séance est un entretien semi-dirigé qui se réalise après chaque séance observée afin de demander à l'enseignant d'évaluer à chaud son activité d'enseignement. L'analyse de ce type d'entretien permet au chercheur d'examiner les objets de savoirs effectivement transmis, et/ou le "savoir enseigné", par l'enseignant. 
Nos résultats seront validés en se référant également aux travaux de Hall (1966) et à son échelle de distances : intime, personnelle, sociale et publique selon deux modes (proche et éloigné). Nous présentons ces différentes distances dans le tableau 3.

Tableau 3 : Echelle des distances interpersonnelles selon Hall (1966)

\begin{tabular}{|l|l|l|}
\hline Distance & \multicolumn{1}{|c|}{ Mode proche } & \multicolumn{1}{c|}{ Mode éloigné } \\
\hline Intime & Corps à corps & De 15 à $40 \mathrm{~cm}$ \\
\hline Personnelle & De 45 à $74 \mathrm{~cm}$ & De 75 à $1.25 \mathrm{~cm}$ \\
\hline Sociale & De 1.25 à $2.10 \mathrm{~m}$ & De $2.10 \mathrm{~m}$ à $3.60 \mathrm{~m}$ \\
\hline Publique & De 3.60 à $7.50 \mathrm{~m}$ & De $7.50 \mathrm{~m}$ et au-delà \\
\hline
\end{tabular}

Nous nous appuyons dans notre analyse sur l'échelle d'ostension modélisée par Salin (Op. Cit), qui est en mesure de nous renseigner sur les différentes formes ostensives déployées ainsi que le degré de dévolution induit par la corporéité de Samar et d'Ali au moment des interactions didactiques (Robert, 2012). Cette modélisation nous permettre de catégoriser la gestualité enseignante (voir Tableau 4).

Tableau 4 : Modélisation de l'échelle des ostensions des savoirs (Robert, 2012)

\begin{tabular}{|c|c|}
\hline Formes ostensives & Commentaires \\
\hline Ostension directe verbale (ODV) & $\begin{array}{l}\text { Le savoir est énoncé et expliqué par } \\
\text { l'enseignant }\end{array}$ \\
\hline $\begin{array}{l}\text { Ostension directe physique partielle } \\
\text { (ODPP) } \\
\text { Ostension directe physique totale } \\
\text { (ODPT) } \\
\text { (Démonstration) }\end{array}$ & $\begin{array}{l}\text { Le savoir est démontré physiquement par } \\
\text { l'enseignant. }\end{array}$ \\
\hline $\begin{array}{l}\text { Ostension physique privée partielle } \\
\text { (OPPP) } \\
\text { Ostension physique privée Totale } \\
\text { (OPPT) } \\
\text { (Manipulation) }\end{array}$ & $\begin{array}{l}\text { Le savoir est montré à l'élève par } \\
\text { l'intermédiaire d'une manipulation de son corps } \\
\text { par l'enseignant. }\end{array}$ \\
\hline $\begin{array}{l}\text { Ostension déguisée (OD) } \\
\text { (Aménagement du milieu) }\end{array}$ & $\begin{array}{l}\text { Le savoir est montré par l'intermédiaire d'une } \\
\text { mise en avant des objets du milieu, d'un } \\
\text { questionnement de l'élève sur ces objets. }\end{array}$ \\
\hline $\begin{array}{l}\text { Non d'ostension (NO) } \\
\text { (Dévolution) }\end{array}$ & $\begin{array}{l}\text { La construction du savoir est sous la } \\
\text { responsabilité de l'élève }\end{array}$ \\
\hline
\end{tabular}

L'analyse qualitative est également complétée par une analyse quantitative dont l'objet est le traitement statistique des données recueillies portant sur la pratique d'enseignement de Samar et Ali. Ainsi, ce traitement statistique a été réalisé à partir du logiciel Sphinx iQ 2 via l'analyse du Khi2. Cet outil d'enquête est susceptible d'apporter une plus-value technique et méthodologique de l'analyse des données. Cette approche méthodologique «mixte» nous permet de mieux cerner l'acte didactique et comprendre les 
ressorts $\mathrm{du}$ fonctionnement didactique de chaque enseignant afin d'en construire deux vignettes didactiques cliniques.

\section{Résultats}

Les résultats présentés dans cet article ont été interprétés à partir des séquences vidéo significatives ${ }^{17}$ tirées d'un visionnage de l'épreuve d'enseignement de nos deux participants que nous avons appelés Samar et Ali. Ces deniers nous ont permis de catégoriser et d'analyser in fine les pratiques ostensives. Ces résultats émanent aussi de l'analyse de l'entretien d'accès au déjà-là décisionnel pour louper le processus combinatoire de leurs pratiques ostensives et proxémiques à partir du déjà-là intentionnel, conceptuel et expérientiel. Notre objectif est de décoder les enjeux manifestés par l'intervention corporelle professionnelle de nos deux enseignants associés et un (ou plusieurs) étudiant (e) s.

\section{Cas de Samar}

Déjà-là décisionnel

Le déjà-là décisionnel du sujet-enseignant se trouve au carrefour du déjà-là expérientiel en référence aux diverses expériences du sujet que cellesci soient d'ordre professionnel ou plus privées, du déjà-là conceptuel qui renvoie au système de représentations, de croyances et de valeurs du sujet et du déjà-là intentionnel qui s'applique aux intentions didactiques du sujet enseignant (Carnus, 2003). La prise en compte de cet ensemble fait partie de « la prise de décision» (Carnus, 2009) au moment de la pratique effective d'enseignement. Au cœur du déjà-là décisionnel, Samar organise son savoir à enseigner et construit du sens en faisant écho à son histoire personnelle, son expérience professionnelle et l'institution dans laquelle elle prend position (Laot, 2009). Samar a l'athlétisme « dans les tripes ». Elle est passionnée par cette spécialité sportive qui correspondait parfaitement à ses centres d'intérêt et ses ambitions : "l'athlétisme c'est le sport que j'admire, c'est lui qui m'a donné la motivation pour atteindre mes objectifs académiques, scientifiques et professionnels ».

Son histoire singulière d'ancienne athlète, d'entraineur fédéral d'athlétisme et d'enseignante d'athlétisme à l'ISSEP de Tunis, souligne un déjà-là expérientiel singulièrement construit dans le plaisir et la fierté de son choix professionnel : «je suis émerveillée par mon parcours sportif et par ma

\footnotetext{
${ }^{17}$ Des unités de sens significatives et saillantes pour la recherche en regard des questions posées. Les captations vidéo cultivent des données relatives au déroulement de la séquence didactique et aux positions assumées par l'enseignant. Elles sont à ce titre fondamental pour La recherche, en ce qu'elles permettent au chercheur de mesurer l'écart entre le discours du sujet et ce qu'il fait, et ainsi de permettre une reconstruction plus fine dans l'après-coup.
} 
carrière ...je suis satisfaite $d u$ chemin que j'ai parcouru $\gg^{18}$. En effet, elle conçoit l'athlétisme comme une discipline muldimensionnelle tout en prenant en considération la spécificité de cette pratique qui s'attache à être « complexe ». Pour cette raison, Samar estime qu' "il faut aller jusqu'au bout avec les étudiants». A ce propos, elle déclare vouloir regrouper à la fois des intentions éducatives et didactiques pour "perfectionner les connaissances qui sont déjà acquises chez les étudiants».

En revenant sur son parcours d'étudiante, Samar approuve que sa formation initiale lui a permise d'avoir les outils pédagogiques et didactiques nécessaires pour gérer la complexité de la situation d'enseignementapprentissage en Athlétisme et d'aider ses étudiants à apprendre et à se préparer à leur métier de futurs enseignants. Ses propos de l'entretien d'accès au déjà-là nous permettent de souligner que sa pratique professionnelle est imprégnée de son vécu sportif antérieur et ses expériences d'athlète confirmée et d'entraîneur qualifiée. A ce propos, Samar précise que : «l'expérience pratique est très enrichissante, elle me donne toujours la chance d'évoluer professionnellement $\gg$. Son désir d'enseigner se manifeste clairement à travers la transmission verbale des savoirs pour aider leurs étudiants à mieux apprendre. Samar éprouve un sentiment de satisfaction de son propre corps qui s'implique dans des enjeux d'enseignement /apprentissage. Dans ce sens, elle illustre ainsi sa pensée : «quand j'enseigne l'athlétisme, je me ressource, je me sens aussi bien satisfaite qu'émerveillée ».

L'impossible à supporter « je ne supportais pas les défaillances des étudiants»

Pour établir une relation didactique avec ses étudiants, Samar partage avec eux en classe des expériences pratiques pour corriger, montrer, démontrer et réguler à des distances variées. Samar semble manifester parfois une « nervosité ressentie » (Ottet, 2016) lors d'une situation imprévue pour parer un mouvement d'étudiant (s) en difficulté technique : "je me sens ... énervée parfois de ne pas tout enseigner à ces futurs spécialistes », "je ne supportais pas les défaillances surtout techniques des étudiants ». Au vu de ces attributs manifestes, elle semble être clouée par l'exécution parfaite du mouvement. Samar n'accepte pas la déformation de l'activité par les étudiants. Pour elle, ils doivent être de vrais spécialistes et de vrais athlètes : "si non pourquoi ils ont choisi cette spécialité ». Elle se trouve face à des étudiants : "qui possèdent des prédisposions qui ne s'alignent pas parfaitement avec les exigences de l'activité ».

\footnotetext{
${ }^{18} \mathrm{Il}$ est à noter que les extraits de verbatim des enseignantes sont présentés en italique et entre guillemets.
} 
Face à cette situation réelle relative à la difficulté d'apprentissage de certains étudiants, Samar n'hésite pas à "proposer de nouvelles situations pédagogiques différentes et des savoirs non prévus » afin que "chacun des étudiants progresse à son rythme ». Samar mobilise une stratégie adaptative régulatrice de l'apprentissage des étudiants en difficulté. Cependant, elle se confronte à un ressenti paradoxal qui la positionne inconfortablement entre l'enseignant (elle-même) entant que «sujet supposé savoir» (Chevallard, 1985) et l'apprenant (ici l'étudiant) en tant que « sujet supposé apprendre » (Carnus, 2015). Samar a du mal à comprendre les difficultés de certains étudiants bien qu'elle dise avoir tout enseigné. Elle se trouve face à « un impossible à supporter », une formule qui est issue de la psychanalyse qui donne à l'inconscient, d'après Miller (2011), son « armature signifiante, la façon dont chacun sur un mode singulier, va condenser et penser ce qui lui arrive ».

En effet, le réel présente toujours un manque en tant qu'il est un impossible à supporter (Lacan, 1977). Il s'agit d'une contingence qui permet de le démontrer. Cette dernière reste l'objet de préoccupation pour l'enseignant qui « peut aller jusqu'à perdre pied » (Blanchard-Laville, 2001). A l'égard de notre étude, nous nous référons à cette notion pour opérer « le dit et le non-dit » de Samar qui se sent : «frustrée » de «ne pas pouvoir donner $d u$ savoir » (Delanoë \& Labridy, 1983), soit «l'impossible à enseigner » tel qu'il a été décrit par Terrisse en 2009. Cet énoncé révèle que son impossible à supporter est lié à l'idée de ne pas réussir à trouver cet équilibre interne et à soutenir sa place d'enseignante (Terrisse \& Carnus, 2009).

\section{Rapport au corps}

L'analyse détaillée des différentes formes ostensives (Salin, 2002) mobilisées par Samar permet de comprendre la mise en scène de son corps dans l'espace de la classe. Au moment de l'épreuve d'enseignement, Samar fait recours à des formes de « distance didactique » (Forest, 2006) pour transmettre les savoirs. Ces formes de « distances didactiques » sont associées à des aspects proxémiques (Hall, 1964,1971). Cela traduit une certaine relation entre l'ostension et la distance qui s'avère ne pas être une simple distance métrique, mais plus tôt porteuse d'une dimension didactique (Brousseau, 1986). Grace à son corps régulateur, Samar produit toutes les actions corporelles nécessaires pour faciliter l'assimilation du savoir auprès de ses étudiants. En effet, Samar fait recours récurrent à son expertise corporelle et à son déjà-là expérientiel s'organisant autour de son expérience personnelle incarnée et de sa connaissance prolongée de l'activité athlétisme. Elle expose un savoir qui aide les étudiants à s'enrôler dans des tâches didactiques de difficulté croissante (Jorro, 2004, 2005). Elle adopte la posture de « régulatrice » en assurant des régulations et des remédiations qui se déclinent 
en plusieurs formes d'aide susceptibles de rendre l'apprentissage plus accessible pour ses étudiants.

En effet, Samar s'engage dans des attitudes non verbales d' « immediacy », c'est-à-dire de «proximité non verbale », qui permettrait l'amélioration de ses compétences à communiquer le savoir en jeu et probablement son efficacité en classe (Thomas, Richmond \& McCroskey, 1994 ; Richmond \& McCroskey, 2004). A cet égard, nos résultats obtenus suite à un visionnage des séquences vidéos des deux épreuves de Samar (E1 et E2) nous ont permis de bien catégoriser et classer toutes les formes ostensives et proxémiques visées par notre étude en vue d'en extraire le sens. Ces résultats obtenus indiquent que Samar fait recours à une gestualité qui se met au service de ses intentions. Focalisée sur les étudiants en difficulté, Samar intervient avant, pendant et après leurs actions motrices. Elle utilise l'ostension directe verbale (Séance $1: 12 \%$ et séance $2: 18.2 \%$ ) pour transmettre les savoirs et les consignes à ses étudiants. Cette intervention est majoritairement associée à une ostension directe physique partielle (Séance 1 : $44 \%$ et Séance $2: 38.6 \%$ ) auprès de quelques étudiants qui sont en difficulté. Samar fait recours à cette pratique ostensive pour démontrer à ses étudiants l'action à réaliser en expliquant verbalement les détails du geste et en engageant son corps dans une démonstration du geste pour montrer à ses étudiants ce qui est réellement demandé. Son intention comme son action sont particulièrement focalisée sur la régulation des apprentissages de ses étudiants.

L'observation de la première séance de Samar nous permet de constater que ses interventions corporelles, telles qu'elles se déploient in situ, créent un espace d'interaction avec les étudiants. Ces derniers adoptent une posture active face aux interventions régulatrices de leur enseignante. Samar a recours massivement à la distance personnelle proche (séance 1: $48 \%$ et séance $2: 34.1 \%$ ) dans les différentes formes d'ostensions qu'elle mette en œuvre. Le graphique1 ci-dessous illustre la combinaison des pratiques ostensives et proxémiques mises en jeu par Samar lors de la première séance d'athlétisme. Il montre la dimension interactive des différentes formes ostensives et proxémiques reflétant ainsi le degré de dévolution consécutif de la gestualité assemblée et/ou combinée par Samar au moment de ses interactions didactiques (Robert, 2012). 
Graphique 1 : Pratiques ostensives et proxémiques de Samar au cours de la 1ère séance

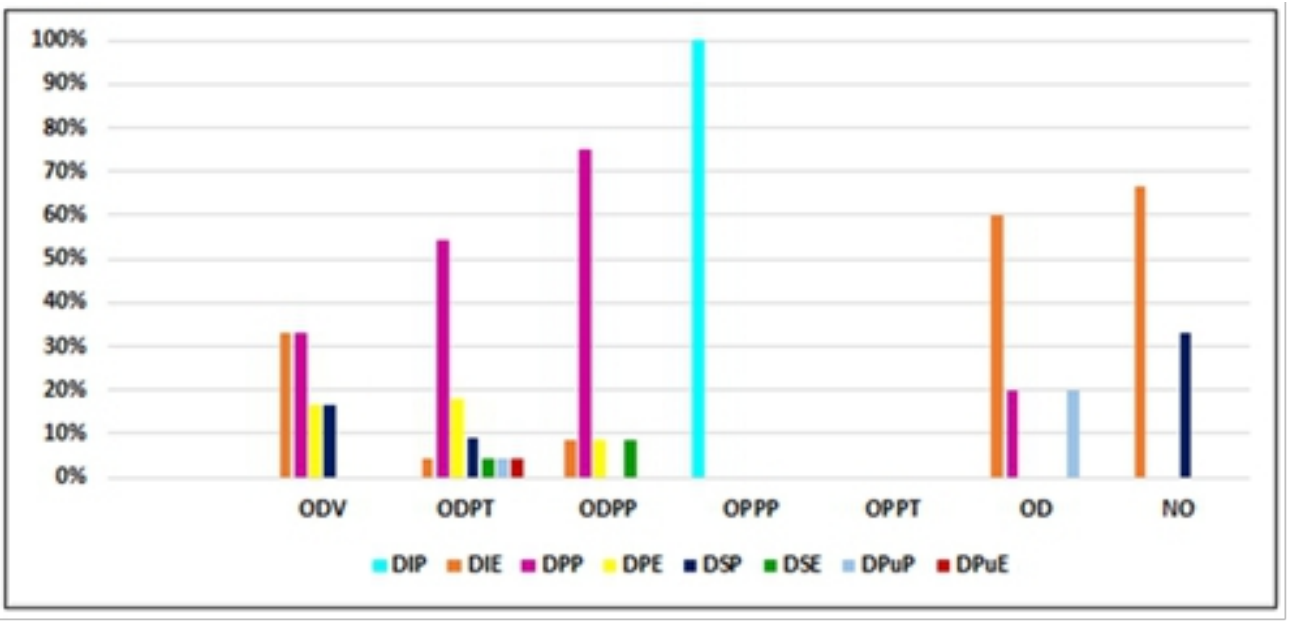

La stratégie de Samar consiste à intervenir auprès des étudiants pour livrer une régulation toute proche de son expertise et un peu distanciée des programmes: "ça m'arrive souvent d'intervenir en m'inspirant de ma carrière en tant qu'athlète pour aider mes étudiants à comprendre le geste ». Son corps est la plupart du temps en mouvement pour soutenir son intervention didactique. Samar s'adresse à tous les étudiants avec son corps « expert » et sa posture "incarnée » d'athlète qui l'habite, pour aider ses étudiants à résoudre les problèmes auxquels ils sont confrontés. Pour Samar, l'objectif essentiel de son intervention didactique corporelle demeure : "un moyen démonstratif qui facilite l'apprentissage de l'étudiant et verbalise mieux le geste ».

Pendant son enseignement, Samar éprouve un sentiment de confort, d'assurance et de satisfaction face à ses étudiants en s'approchant d'eux pour démonter, réguler et corriger. En occupant une distance intime proche (100\%), elle ne peut pas s'empêcher de démontrer : "je trouve un plaisir quand je fais une démonstration devant mes étudiants, c'est comme ça qu'ils réussissent à mémoriser un geste correctement, ça me rassure doublement, du résultat de leur action et de l'efficacité de mon intervention, du coup, ça va, je me sens satisfaite ». A travers ce recours récurrent à la démonstration gestuelle combinée à une distance intime proche, Samar s'expose au regard de ses étudiants, montre son expertise corporelle et incarne un statut narcissique de «Sujet supposé Savoir » ${ }^{19}$ (Lacan, 1968 -2001) cité par Chevallard, 1985).

${ }^{19}$ Le « Sujet Supposé Savoir » est outil conceptuel pour analyser la manière singulière dont chaque enseignant occupe la fonction de détenir et de transmettre le savoir. la fonction symbolique de « Sujet supposé Savoir » est déterminée institutionnellement ; La recherche en 
D'autres formes proxémiques ont été également repérées à partir de l'observation et l'analyse de la pratique effective de Samar, qui consistent à laisser agir et à donner par la suite des remédiations et/ou régulations individualisées à l'étudiant par la manipulation de son corps.

Samar envisage une ostension physique privée partielle (séance $1: 4 \%$ et séance $2: 13.6 \%$ ) et totale (séance $2: 9.1 \%$ ) du début jusqu'à la fin de l'action demandée pour que l'étudiant se positionne correctement et assimile l'action sollicitée. il s'agit de cibler une partie bien particulière du corps de l'étudiant afin d'en apporter les corrections nécessaires. Pour Samar, ces ostensions physiques privées servent de repères aux étudiants en leur permettant de ressentir le mouvement pour ensuite ajuster leurs actions. Ainsi, nous notons que les effets de ces deux formes d'ostension privées, totales et partielles, sur les résultats d'apprentissage deviennent plus significatifs auprès de la majorité des étudiants. De ce fait, nous pouvons soutenir le point de vue de Durant (1996) selon lequel « la singularité et la créativité de l'action du maitre sont très vites identifiées par les élèves ». Samar se sert de son corps « exhibé » pour embellir son intervention didactique et laisser ainsi «sa signature professionnelle »(Blanchard-Laville, 2000). Son corps est tout le temps animé par une dynamique d'un désir d'incorporer le savoir et de verbaliser le corps, un corps «didactisé » assurant ainsi des « fonctions des savoirs » variées (Simon \& Schmehl-Postaï, 2010).

Le graphique 2 ci-dessous présente les formes ostensives utilisées par Samar lors de la deuxième séance d'athlétisme ainsi que les différentes distances proxémiques y sont combinées. Elle corrige presque chaque étudiant à part en pénétrant dans son espace didactique d'apprentissage d'une façon singulière et ciblée (OPPP : 13.6\%). Samar apporte les mêmes régulations qu'elle les maitrise parfaitement à partir d'un positionnement spatial significatif, d'une « zone de rencontre » (Baeza-Carminatti, 2007), lieu d'interaction, de construction et de transmission de savoirs.

didactique clinique s'attache à préciser la modalité singulière pour un enseignant d'habiter cette fonction (Carnus \&Terrisse, 2013, p143). 
Graphique 2 : Pratiques ostensives et proxémiques de Samar au cours de la 2ème séance

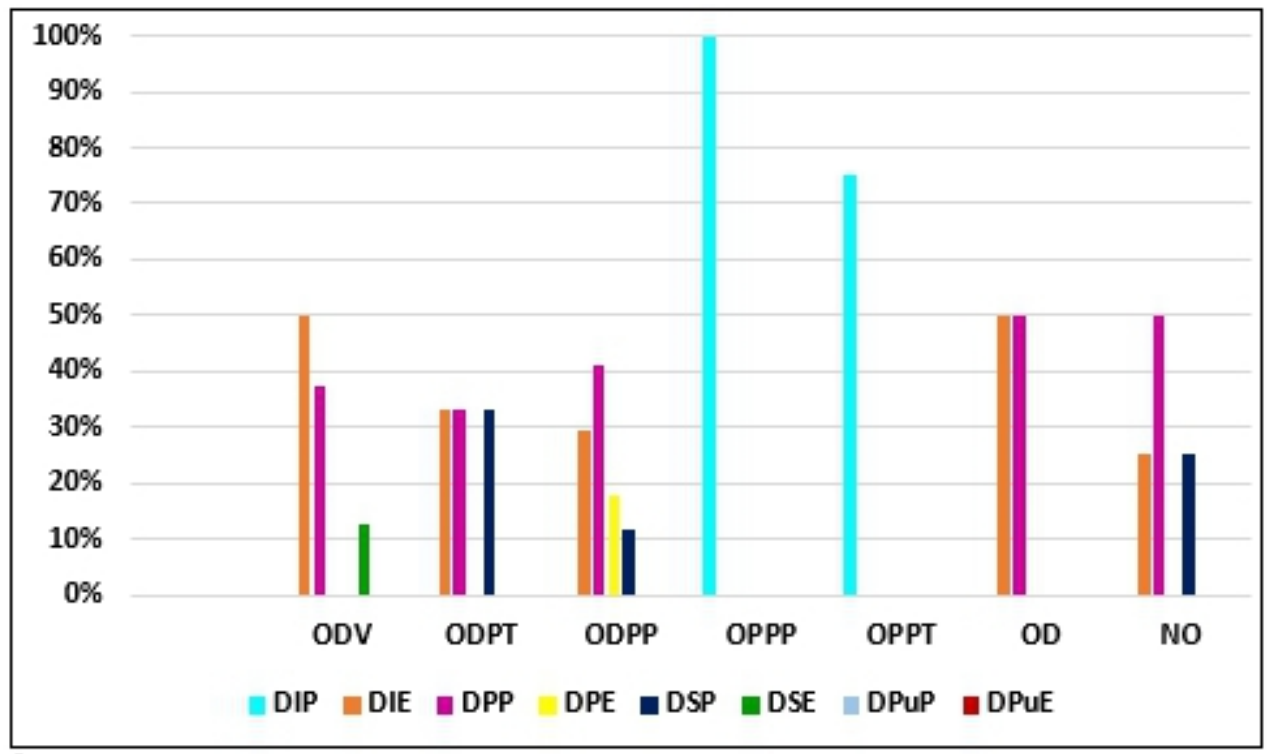

Cet espace que Samar construit consciemment, et parfois inconsciemment, englobe différentes formes proxémiques. En passant de DPP (34.1\%) vers une DIE (27.3\%), elle met en jeu une DIP (20.5\%) pour accompagner les étudiants avec une grande implication corporelle voire même « un déjà-là proxémique ». En effet « où les corps sont mis en jeu, une des particularités est de se rapprocher voire de toucher l'autre pour répondre aux problèmes » (Carminatti \& Carnus, 2019). L'observation et l'analyse des épreuves d'enseignement de Samar nous ont permis de constater que plus l'étudiant se trouve en difficulté d'apprendre dans une situation donnée, plus Samar est impliquée corporellement pour lui communiquer le savoir manquant, et ce, à travers des formes ostensives et proxémiques combinées. En effet, la pratique enseignante de Samar s'apparente à un processus combinatoire entre l'ostension et la distance proxémique mises en œuvre dont la relation, selon les résultats statistiques relatifs à l'outil Khi2, s'avère très significative $(\mathrm{S} 1$ : Khi2 $=79.63$ et $\mathrm{S} 2$ : Khi2 = 53,17). Samar s'engage dans une activité régulatrice afin de répondre aux attentes des étudiants, de proposer une pertinence pour faire fonctionner le groupe (Baeza-Carminatti \& Nourrit, 2009) et d'être efficace.

\section{Cas d'Ali}

\section{Déjà - la décisionnel}

Ali entretient un rapport aux savoirs qui s'actualise au fur et à mesure de son interaction avec ses étudiants en situation de classe. Toutefois il définit la nature du savoir qu'il veut transmettre en termes de compétences à acquérir. 
Pour lui, son rôle consiste à favoriser un climat didactique où ses étudiants réussissent à construire leurs savoirs via les connaissances qu'ils ont déjà acquises : « il n'y a de cours totalement préparé. C'est un cours interactif entre moi et mes étudiants, mon rôle est de perfectionner ce qu'ils ont déjà appris parce que pour choisir une spécialité, il faut avoir déjà des compétences et des habiletés quand même ». Ali considère les étudiants comme étant des partenaires essentiels au sein de cette « mise en œuvre » didactique.

Pour Ali, les étudiants ont la responsabilité de gérer leurs acquis et préconiser un nouvel apprentissage : "il faut que l'étudiant essaie d'acquérir la discipline tout seul ». Conformément à cette conception d'apprentissage focalisée sur l'étudiant, Ali soutient la thèse, adoptée par plusieurs chercheurs, selon laquelle «l'organisation, l'activation et la disponibilité des connaissances antérieures influencent l'apprentissage de nouvelles connaissances, ainsi que la capacité à réutiliser ces notions dans des contextes variés » (Brandsford, Vye, Adams \& Perfetto, 1989). Ali cherche à faire passer ses pratiques enseignantes centrées sur l'enseignant (teacher-centred) vers des pratiques centrées sur l'étudiant (student-centred), une stratégie confirmée par Kember en 1997.

L'étude de cas d'Ali montre que sa pratique enseignante renvoie principalement à une référence personnelle en lien avec son histoire intime sur laquelle il s'appuie pour répondre aux besoins et aux attentes des étudiants. Il s'agit bien de son savoir pratique, son parcours d'expertise et de son rapport personnel à sa spécialité sportive. Son désir d'enseigner anime sa pratique : «je suis émerveillé par ma carrière », «l'athlétisme c'est mon bonheur, toute ma vie ». L'athlétisme émane d'un choix personnel du métier qui lui facilite « le passage du savoir à enseigner au savoir enseigné » (Chevallard, 1991). Il s'engage à transmettre «des savoirs faire et des savoir y faire » (BuznicBourgeacq, Terrisse \& Lestel, 2008) pour amener ses étudiants à mobiliser leurs acquis techniques à bon escient et agir adéquatement dans une situation d'apprentissage donnée.

Ali se trouve animé aussi par le poids institutionnel de sa formation initiale pratique et théorique, par les différentes formations spécialisées ainsi que son statut particulier d'enseignant -chercheur qui lui garantit une formation de qualité. Ali souligne un rapport à l'athlétisme profond et ancré dans son histoire identitaire même : «l'athlétisme est une partie de moi ». S'identifier en tant qu'expert dans son domaine révèle un sentiment de maitrise et de compétence qui se légitime à travers sa longue carrière sportive et professionnelle d'enseignant-chercheur. Pour lui, son rôle principal demeure «d'actualiser sa pratique enseignante» et d'améliorer «les conditions favorisant l'apprentissage des étudiants » (Deschryver \& Lameul, 2016). 


\section{L'impossible à supporter}

En prenant en compte le positionnement subjectif de Ali, nous constatons qu'il met en exergue un impossible à supporter qui le divise entre ce qu'il avait l'intention d'enseigner et ce qu'il ne peut s'empêcher de faire. Ainsi, Ali ne supporte pas le non savoir de ses étudiants : «ce que je n'accepterais pas c'est que les étudiants font semblant de ne pas comprendre ». Pour lui sa mission se cantonne à construire des situations d'apprentissages didactisées. En échange, ses étudiants doivent participer activement et d'une façon «autonome et responsable » à leur apprentissage : «être des partenaires à part entière » de l'acte didactique. Bien entendu, ces étudiants sont censés travailler de façon autonome puisqu'ils sont censés maîtriser une bonne partie de cette discipline sportive : «ils ont choisi cette activité en tant que spécialistes et ils sont censés la maitriser, mon rôle est de leur fournir un nouveau savoir et valider leurs compétences et contrôler leur progression ».

Toutefois, Ali se trouve obligé d'inciter ses étudiants à prendre de l'initiative et à assumer leur part de responsabilité dans l'apprentissage : «je demande aux étudiants de travailler à l'avance les progressions pédagogiques des éléments techniques et nous essayons de les aborder en classe ». Ali ne comprend pas pourquoi ses étudiants n'assument pas cette responsabilité surtout que le savoir est devenu accessible en dehors de la classe. En effet, il exprime son intention d'établir des compromis, parfois de compromissions élaborées entre concessions et renoncements (Carnus, 2002) afin d'aider efficacement les étudiants à s'approprier des savoirs et maîtriser suffisamment les techniques de la discipline. Il précise que sa relation pédagogique avec eux serait une condition fondamentale pour qu'ils apprennent. Pour lui : «le savoir, le savoir-faire et le savoir-être » constituent trois composantes essentielles et interdépendantes de la compétence. De même, Ali déclare utiliser "la critique constructive » dans son enseignement de l'athlétisme et inculque à ses étudiants l'esprit d'équipe où ils peuvent développer leur pensée critique et réflexive. Ali évoque l'autonomie que les étudiants devraient gagner à l'université et leur maturité attendue par rapport à l'enseignement de la spécialité.

\section{$\underline{\text { Rapport au corps }}$}

Du point de vue du rapport au corps d'Ali, nous pouvons mettre en avant que ses pratiques corporelles traduisent un savoir en « je » (Carnus, 2009) qui affecte le processus de transposition didactique interne. En effet Ali fait usage d'un savoir issu «d'une histoire personnelle de pratiquant » 
(Loison, 2009). Cette « conversion didactique ${ }^{20}$ (Buznic-Bourgeacq, 2005) est singulière et plus ou moins «maitrisée et conscientisée » (Jourdon, 2005 ; Buznic- Bourgeacq, 2009 ; Léal, 2012). Ali évoque un sentiment de respect et d'amour envers ses étudiants qui les considère comme : «des vrais acteurs ». Il met leur participation au cœur du processus d'enseignement : «l'étudiant c'est lui l'enseignant en ce moment, moi je suis le médiateur de la séance », « d'ailleurs, l'enseignant n'est pas la seule source d'informations ».

Ali conçoit l'enseignement universitaire comme une pratique qui a trait à l'importance du lien entre la participation active des étudiants à travers la mobilisation de leurs connaissances et l'accompagnement de l'enseignant à travers ses "ressources subjectives en vue d'accompagner l'apprentissage » de l'enseignant (Touboul, Carnus, Terrisse, 2012). De plus, il considère qu'il doit être à l'origine de l'interaction et de l'action des étudiants dans leurs cours pratiques afin de les amener à se construire de façon autonome (Baillet, Evi Belsack \& Uyttebrouck, 2019, p.140).

En classe, Ali accompagne d'une façon permanence ses étudiants en impliquant partiellement son corps pour assurer une meilleure transmission des enjeux de savoir à travers des situations d'apprentissage. En effet, il interagit avec eux en privilégiant essentiellement l'ostension directe verbale (E1 : $29.3 \%$ et E2 : $35.6 \%$ ) pour transmettre des consignes, leur faciliter leurs apprentissages et les inciter à apprendre. Son enseignement revêt principalement un caractère verbal du fait qu'il est axé sur la parole, les explications et les descriptions techniques verbales.

Ali transmet un savoir « universitaire problématisé nécessitant une acculturation disciplinaire » (Baillet et al., op. Cit, p137). Par «Acculturation disciplinaire aux savoirs », nous entendons au sens de Baillet et ses collaborateurs (Op. Cit. p. 137) ${ }^{21}$, «l'identification et la compréhension des langages, méthodes et cadres d'analyse spécifiques à une discipline ». Ali confirme ce propos ainsi : "à ce niveau d'études universitaires, ces futurs spécialistes d'athlétismes sont censés connaître la discipline "athlétisme" et les méthodes d'entraînement ». Il affirme qu' "avec l'âge et l'expérience, nous expliquons le geste verbalement plutôt que nous le démontrons d'une manière corporelle ». Toutefois, cet échange verbal dans l'espace didactique de la classe s'opère en lien avec une variété d'aspects proxémiques. Comme

${ }^{20}$ La conversion didactique permet de revisiter la référence des savoirs scolaires en identifiant la part du sujet enseignant qui les fonde dans leur transmission effective (Carnus et Terrisse, 2013, p.141).

${ }^{21}$ Nous reprenons la définition de (Baillet et al. op. Cit, p136) des « savoirs problématisés » qui les présentent comme «des savoirs qui font référence à la fois au contexte (questions, problèmes) à l'origine des savoirs, aux conditions dans lesquelles les recherches sont menées, aux contraintes (intellectuelles, matérielles) auxquelles les chercheurs sont confrontés, à leurs questionnements, aux débats que les recherches ont fait émerger », bref à ce que Rey et ses collaborateurs appellent les «pratiques-sources » (Rey et al., 2003). 
l'indique le graphique 3 ci-dessous, nous remarquons qu'Ali ne se positionne pas dans un endroit précis. Il transmet des consignes dans une dynamique interactive de classe, selon des types de distances interpersonnelles variées. Cette mise à distance, principalement personnelle d'enseignement verbal, se fait en mode sociale éloigné (42.2\%) pour assurer la transmission de conseils individualisés selon une conception adaptable puisqu'elle varie selon le contexte d'enseignement (Romainville, 1998) et évolutive (Kugel, 1993).

Graphique 3 : Pratiques ostensives et proxémiques de Ali au cours de la 1ère séance

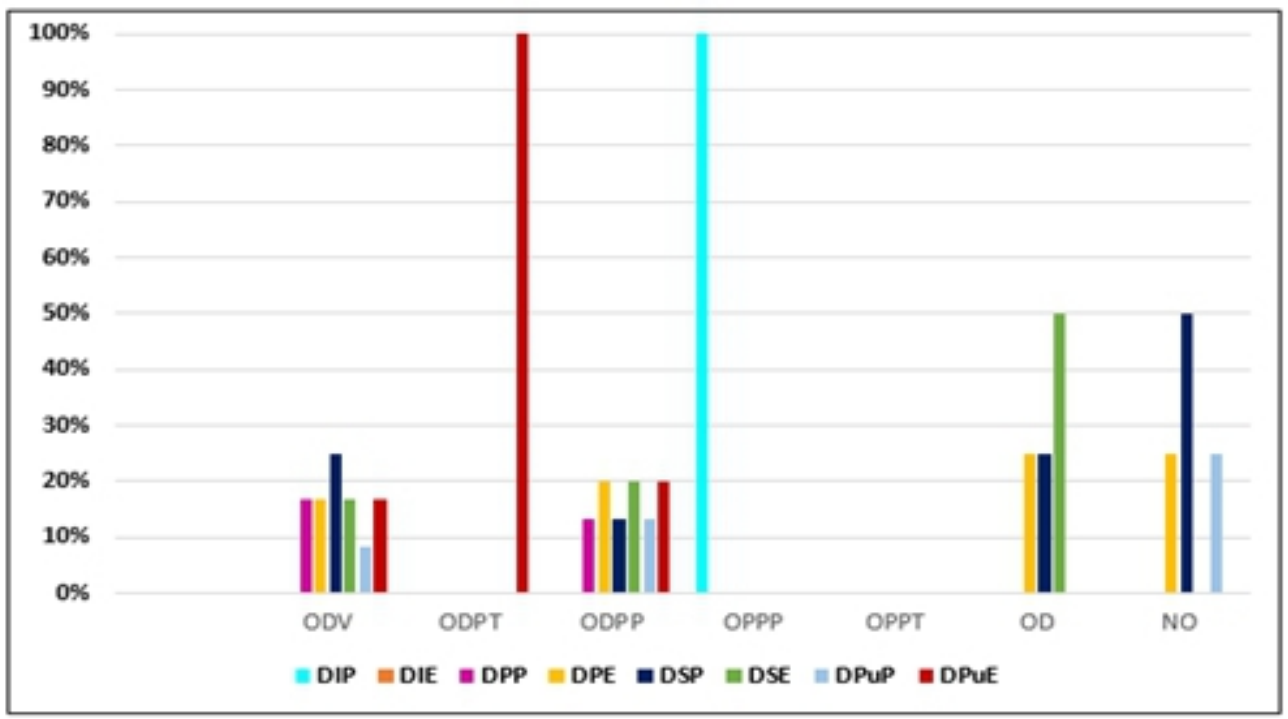

Une fois les consignes sont procurées, Ali se retire pour laisser les étudiants construire le savoir individuellement ou en petits groupes. Il utilise la forme de non ostension (Séance 1:9.8\% et Séance 2: 15.6\%) pour responsabiliser ses étudiants, les engager dans l'adoption d'une posture réflexive et développer leur autonomie. La mise en scène corporelle d'Ali s'attache à être porteuse du fait qu'elle représente un moyen " pour montrer, démontrer, corriger à des distances variées » (Magali, \& Geneviève, 2012). Ali fait recours à l'ostension directe physique partielle (Séance $1: 28 \%$ et Séance $2: 26 \%$ ) pour adapter ses intervenions didactiques aux exigences techniques du geste. De plus, il n'utilise que très rarement la manipulation sous forme d'ostension physique privée partielle (Séance $1: 12.2 \%$ et Séance $2: 4.4 \%$ ). Cette forme d'ostension ciblée aide les étudiants à comprendre le geste technique et ressentir des positions et des mouvements bien précis.

Ce savoir «expert »(Joshua, 1996) reflète une connaissance personnelle très spécifique et profonde de sa spécialité. Ali sait prendre des décisions rapides et efficaces pour affiner les habilités de ses étudiants en utilisant des gestes de démonstration précis : «j'essaye de minimiser mes intervenions pour laisser l'étudiant réagir face à une situation problème mais 
si ça ne fonctionne pas j'interviens tout de suite pour exécuter le geste qu'il faut pour l'aider à surmonter cet obstacle ». Ali envisage choisir ses mouvements selon des procédures ostensives déguisées (Séance 1:9.8\% et Séance $2: 15.6 \%$ ) à travers l'aménagement du dispositif didactique (entre autres matériel), auquel les étudiants participent en assumant plusieurs rôles (Léal, 2006) et en s'adaptant en fonction du contexte dans lequel ils évoluent : "Je vais même proposer à mes étudiants un matériel et un équipement spécifique à la discipline, eux ils savent quoi faire avec ce matériel ». Les résultats présentés dans le graphique 4 ci-dessous, montrent que la distance interpersonnelle entre les protagonistes de la situation d'enseignementapprentissage peut influencer la qualité de la relation éducative. D'un point de vue Statistique, nous notons une relation très significative entre l'ostension et la distance proxémique pendant l'épreuve interactive d'enseignement (S1 : Khi2 $=56.85$ et $\mathrm{S} 2:$ Khi2 $=62.06$ ).

Dans ce cadre, Ali prend un certain recul pour ne pas se laisser entrainer dans le jeu de l'étudiant en occupant massivement une distance sociale éloignée (Séance 1: $17.1 \%$ et Séance $2: 42.2 \%$ )). Il se positionne dans un endroit lui permettant d'échanger verbalement avec ses étudiants tout en étant distant d'eux : "je préfère être un peu distant par rapport à eux tout en gardant le contrôle de la classe et encourageant les étudiants à s'engager dans l'activité ». Cette distance est révélatrice d'une position didactique assumée vis-à-vis l'apprentissage des étudiants puisqu'elle leur permet ainsi de construire activement et d'une façon relativement autonome des savoirs disciplinaires problématisés. Ce qui est en mesure d'assurer le saut qualitatif des savoirs appris par les étudiants dans un milieu didactique qui ne risque pas de confondre le rôle de l'enseignant à l'activité d'apprentissage de l'étudiant. 
Graphique 4 : Pratiques ostensives et proxémiques d'Ali au cours de la 2ème séance

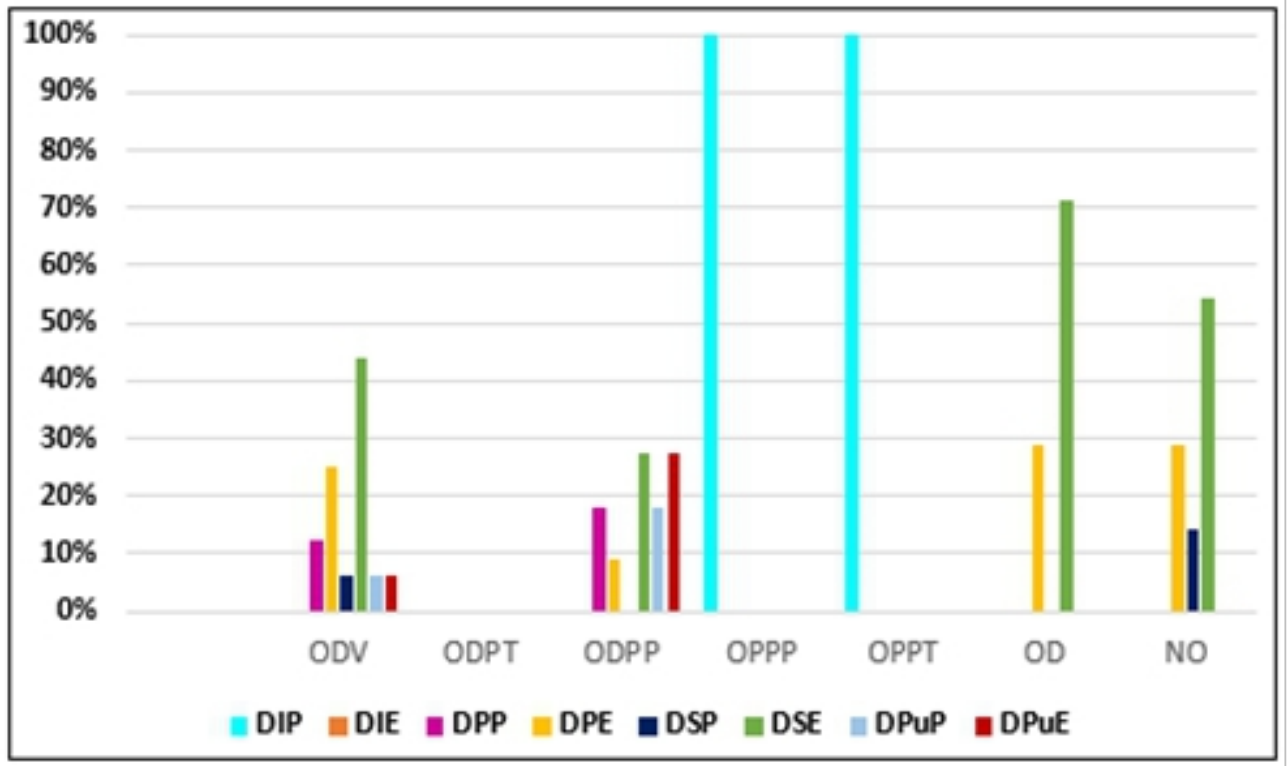

Pour Ali, la séance d'athlétisme ne s'improvise pas. Elle se construit et/ou se co-construit d'une façon structurée, adaptative et évolutive pour que ses étudiants, ce « public précis » acquiert des savoirs, des savoirs faire voire même des «savoirs y faire» (Terrisse, 2009). Pour ce faire, Ali incite ses étudiants à s'engager dans l'activité de construction de leur propre apprentissage puisqu'il estime que "rien n'est donné, tout est construit » (Bachelard, 1938). Dans cette logique de compétence professionnelle, les variations des pratiques ostensives, proxémiques et les enjeux du corps qui en découlent occupent une place assez importante dans l'enseignement d'Ali.

\section{Etude de cas rapprochés}

\section{Similitudes}

Les similitudes renvoient à des traits communs entre les deux enseignants associés. En effet, leurs enseignements sont influencés par un « déjà-là expérientiel sportif » et une « identité de chercheur » (Baillet et al., Op. Cit, p.140). Leurs pratiques enseignantes révèlent « une mise en œuvre de l'activité dans une situation donnée, incluant la planification, les contraintes, les choix et les prises de décision »(Vinatier \& Altet, 2008). En les interrogeant sur leur rapport au savoir, Samar et Ali signalent qu'ils se réfèrent aux programmes officiels et au projet d'établissement pour fonder leurs savoirs et construire leurs enseignements. Cependant, ils n'hésitent pas, chacun à sa manière, de se détacher des documents institutionnels en se basant sur leurs rapports personnels informels au savoir. Faisant écho à leur vécu sportif et leur méta-connaissance sur cette discipline sportive et ce domaine de 
pratique, qui est l'Athlétisme, Ali et Samar mettent en jeu un corps «porteur de savoir-faire didactisé »(Jourdan, 2006) qui se déploie dans l'espace didactique et social de la classe tout en étant animé par un rapport au savoir « actif et créatif » (Beillerot, 1996).

Ce rapport au savoir s'avère ainsi « le processus par lequel un sujet, à partir de savoirs acquis, produit de nouveaux savoirs singuliers lui permettant de penser, de transformer et de sentir le monde naturel et social » (Beillerot, op. Cit). Ce rapport au savoir créateur du sens caractérise la pratique enseignante des deux enseignants observés qui ont tendance à «convertir » (Buznic-Bourgeacq, 2009) leurs expériences sportives et professionnelles en contenu d'enseignement et à transmettre un savoir expérientiel singulier.

De même, l'analyse de l'épreuve d'enseignement de Samar et Ali permettent d'identifier des attitudes corporelles, des gestes professionnels et des conceptions d'enseignement qui correspondent à leurs intentions déclarées lors de l'entretien d'accès au déjà-là, à l'égard des savoirs à enseigner, de leurs conceptions d'enseignement et de leurs rapports au savoir et à la discipline athlétisme. Ce constat résultant de la triangulation des données (Van Der Maren, 1996) confirme le poids de l'histoire singulière du sujet, qui contribue «à façonner chez eux un rapport personnel à l'activité physique » (Loizon, 2009). Leurs choix didactiques, leurs pratiques effectives, leurs stratégies proxémiques et ostensives et leurs modalités d'emploi de distance didactique permettent de rendre compte de ce qu'ils sont et de ce qu'ils pensent en classe. Au moment de leur épreuve interactive, Ali et Samar favorisent des stratégies didactiques «sans nécessairement l'avoir décidé ni en être conscients ». Ils montrent un geste technique de différentes manières et via diverses méthodes pour faciliter l'apprentissage à l'étudiant.

Cette dimension ostensive renvoie à un «contrat d'ostension » (Brousseau, 1996). Ce dernier repose sur « une hypothèse épistémologique empiriste et réaliste qui arrange apparemment les deux parties » (Brousseau, 1997). Ali et Samar sont tous les deux inscris dans la logique intentionnelle de ce contrat, mais chacun à sa manière en se référant à son arrière-plan qui « détermine ses jugements, ses concepts et ses réactions » (Wittgenstein, 1994). Durant leurs épreuves interactives, Ali et Samar construisent à leurs insu des distances proxémiques qui renforcent leurs « déjà-là » (Carnus, 2001, 2003). Ce dernier reflète « une nouvelle facette révélatrice d'un inconscient proxémique » (Carminatti \& Carnus, 2019) qui influence les stratégies didactiques des deux enseignants. Ce langage corporel particulier et cet espace communicationnel significatif dépassant la simple répartition spatiale des sujets, nous fait penser à un nouveau filtre de l'action didactique présenté par Carminatti et Carnus (Op. Cit) sous l'appellation de « déjà-là proxémique ». Ce dernier se présente comme un outil heuristique conduisant à interroger les 
effets d'un «inconscient proxémique » sur les savoirs en jeu lors des rencontres entre enseignants et apprenants.

\section{Différences}

Contre toute attente, les résultats qui ont été présentés révèlent également une différence entre la corporéité féminine et celle masculine au niveau des formes proxémiques et des distances mises utilisées lors de la transmission des savoirs. Du côté de Samar, son intervention didactique s'annonce particulièrement régulatrice. Elle déclare qu'elle préfère être toute proche de ses étudiants pour pouvoir intervenir et corriger, motiver et susciter leurs désirs d'apprendre : «je vais démontrer pour les aider à imaginer le geste ...et être toute proche d'eux pour ajuster leurs actions ». Par contre, Ali choisit de se positionner à une distance assez éloignée au moment de sa pratique effective pour que ses étudiants arrivent, tous seuls, à construire leurs propres savoirs et apprendre: «je vais être là mais je vais éviter les démonstrations, il faut que l'étudiant essaie d'acquérir la discipline tout seul».

A travers l'analyse fine du déjà-là intentionnel, conceptuel et expérientiel, nous avons pu identifier un modèle de corps professionnel féminin émancipé, exhibé et impliqué pleinement dans l'activité d'enseignement, un corps «didactisé » du fait qu'il se veut « régulateur » de la situation de transmission-appropriation de savoir. Ce corps régulateur est soutenu par une conception identitaire du corps, un rapport intime au corps et une image d'un corps acteur «senti », « vécu » et «verbalisé » (Jourdan, 2005). Cependant, le corps professionnel masculin est partiellement impliqué, distant et semble être « rationnel ». D'où un rapport distant au corps chez Ali révélateur d'un mode d'engagement masculin faiblement « régulateur», fortement «techniciste » cherchant à dévoluer le savoir à l'étudiant malgré l'incertitude du résultat relatif à son apprentissage réel.

\section{Discussion et conclusion}

$\mathrm{Au}$ terme de cet article nous précisons que les résultats sont issus de l'analyse des deux premiers temps de recueil et d'analyse des données de la méthodologie didactique clinique. Ces deux temps sont relatifs au déjà-là et à l'épreuve d'enseignement de nos deux enseignants universitaires expérimentés et spécialistes d'athlétisme, intervenant dans la formation pratique en STAPS. Ainsi, nous estimons que l'acte d'enseignement dans la formation pratique en STAPS est façonné par le corps (Sghaier et al., 2017; Ben Jomâa et al., 2017). Ce corps intervenant, dans la profession enseignante, diffère selon qu'il est féminin ou masculin. Cette différence se manifeste particulièrement à travers des représentations et des conceptions différentes du corps, des attributions de rôles didactiques différentes et des 
positionnements didactiques et stratégiques disparates à l'égard de l'enjeu du savoir.

A l'issue de cette étude, nous faisons le constat qu'au moment de l'apprentissage Ali invoque une conception «fixiste » ${ }^{22}$ des technique sportives (Vigarello, 1988) basée sur le guidage technique (Bouthier, 1986 ; Andrieu \& Bourgeois, 2004 ; Altet, 1994). Par contre, Samar fait recours à une conception « régulatrice» (Boudard, 2011) fondée sur une stratégie de réorientation des résultats d'apprentissage des étudiants (Vial, 2001) et sur des «gestes d'aide à l'étude » (Chevallard, 1999). Chacun de nos deux enseignants choisit sa propre entrée dans l'activité et son propre mode d'enseignement, à travers un corps « didactisé » qui représente à la fois le contenu et le contenant de l'objet de savoir (Ben Jomâa et al., 2018).

Nous soutenons l'idée que chaque enseignant consulte une référence qui est en mesure de donner du sens à son enseignement. Ce constat trouve appui dans les travaux de Terrisse (2001) qui affirme que « le savoir transmis par l'enseignant se réfère le plus souvent à un déjà-là ». Au moment de leur pratique effective, Samar et Ali mettent en œuvre des pratiques ostensive variées qui mobilisent des rapports au(x) savoir(s) et répercutent également des enjeux de savoir (Carnus \& Robert, 2013). La combinaison de ces différentes formes ostensives pourrait donc révéler le rapport au savoir des enseignants d'EPS, soit leur propre façon d'appréhender et de transmettre le savoir (Sghaier et al., 2019).

\section{Limites de la recherche}

Nous précisons que nos résultats portent uniquement sur deux études de cas, raison pour laquelle nous n'avons pas l'intention de prétendre à la généralisation desdits résultats. Cependant, nous pourrons, à partir de l'effet de « commulativité des résultats » (Carnus \& Terrisse, 2013), penser à produire des invariants théoriques (analyseurs, concepts etc.) applicables sur d'autres enseignants universitaires expérimentés dans d'autres champs disciplinaires. L'analyse didactique clinique des ressorts de l'acte d'enseignement nous conduit à apporter des éléments de compréhension et de clarté relativement intelligibles permettant de penser la place du corps de l'enseignant en classe d'une manière plus approfondie. Selon « le déjà-là proxémique » (Carminatti \& Carnus, op. Cit), «l'épistémologie pratique » (sensevy, 2007) et l'identité professionnelle de l'enseignant et/ou de l'enseignante, un «contrat différentiel d'ostension» (Brousseau, 1996) s'établit et un rapport « différentiel » au corps se mobilise afin d'organiser la forme et le contenu de l'enseignement.

${ }^{22}$ Il s'agit d'une conception fixée sur l'efficacité technique, la spécialisation sportive et «l'engagement compétitif » (Defrance, 2006). 
Ce rapport « différentiel » au corps (masculin vs féminin) se déploie sur la scène didactique à travers des espaces, des distances, des positions, des codes, des attitudes corporelles et des rôles différents qui en disent long sur le corps et la culture disciplinaire dans laquelle s'inscrit culturellement le corps de l'enseignant et celui de l'apprenant. Le corps est «parlant», « signifiant» d'un « signifié » qui réclame bruyamment en coulisse son désir à exprimer ce qui ne va pas...Un champ d'étude qui mérite d'être approfondi et confronté à d'autres construits dans d'autres temporalités et contextes disciplinaires universitaires.

\section{References:}

1. Allal, L. \& Mottier-Lopez, L. (2007). Régulation des apprentissages en situation scolaire et en formation. Bruxelles : De Boeck.

2. Altet, M. (1994). La formation professionnelle des enseignants. Paris : PUF.

3. Amade-Escot, C. (1991). Caractérisation de la formation didactique initiale des enseignants d'Education Physique et évaluation de ses retombées sur les compétences professionnelles. Thèse de doctorat non publiée, Université Toulouse III.

4. Amade-Escot, C. (2002). Etude du travail de l'enseignant d'éducation physique dans la classe : contribution des recherches didactiques à l'analyse des pratiques effectives. In J.F. Marcel (Ed.). Les Sciences de l'Éducation: des recherches, une discipline (pp. 53-78). Paris: L'Harmattan, Savoir et Formation.

5. Andrieu, B. \& Bourgeois, I. (2004). Les interactions langagières tuteur/élèves en travaux personnels encadrés. Aster, 38, 69-90.

6. Bachelard, G. (1938). La formation de l'esprit scientifique. Paris: Vrin.

7. Baeza-Carminatti, N. (2007). La zone de rencontre en Éducation Physique et Sportive: lieu d'interaction, de construction et de transmission des savoirs. Thèse de doctorat non publiée. Université d'Orléans, France.

8. Baeza-Carminatti, N. \& Nourrit, D. (2009). La zone de rencontre en Éducation Physique et Sportive : un espace de construction identitaire. Sciences \& Motricité, 3(68), 51-71.

9. Baillet, D., Belsack, E. \& Uyttebrouk, E. (20019). De l'écart entre rapport au savoir de l'enseignant-chercheur et rapport au savoir des étudiants à la construction d'une formation. In M.-F. Carnus, D. Baillet, G. Therriault \& V. Vincent (Dir.). Rapport au(x) savoir(s) et formation des enseignants. Un dialogue nécessaire et fructueux (pp.133-149). Bruxelles : Editions De Boeck Supérieur. 
10. Bardin, L. (1998). L'analyse de contenu. Paris : Presses Universitaires de France.

11. Beillerot, J. (1996). Désir, désir de savoir, désir d'apprendre. In J. Beillerot, C. Blanchard-Laville \& N. Mosconi (1996). Pour une clinique du rapport au savoir (pp.51-74). Paris : L'Harmattan.

12. Ben Jomaa, H. (2009). L'expertise dans l'enseignement de l'EPS : Analyse didactique clinique de quatre professeurs. Cotutelle de Thèse de doctorat. Université Paul Sabatier. Toulouse III (France), Université de la Manouba (Tunisie).

13. Ben Jomaa, H. \& Sghaier, D. (2017). The Effect of the Relationship to Knowledge of Physical Education Teachers on the Ostensive Forms in Swimming. European Journal of Educational Sciences, 4(4), 24 -36.

14. Ben Jomaa, H., Abdelkefi, H., Chihi, H., Majdoub, S. \& Kpazai, G. (2018). A Clinical Didactics Analysis of the Use of Proxemics Forms in the Teaching-Learning Process of Sports and Physical Education Setting: A Case Study in Tunisia. International Journal of Learning, Teaching and Educational Research, 17(1), 155-165.

15. Ben Jomaa, H. (2019). Genre professionnel et rapport au corps féminin/masculin en didactique de l'éducation physique et sportive : deux études de cas en Tunisie. In M. Lachhab, H. Bedhioufi, S. Mnedla, H. Ben Jomâa, Z. Zerai \& C. Fitouri. (Coordination). La pratique sportive à l'épreuve $d u$ genre ( $\mathrm{p}$ 269). Tunis: Série Recherches.

16. Blanchard-Laville, C. (2000). L'analyse des pratiques professionnelles. Paris : Harmattan.

17. Blanchard-Laville, C. (2001). Les enseignants entre plaisir et souffrance. Paris : PUF.

18. Bransford, J. D., Vye, N. J., Adams, L. T. \& Perfetto, G. A. (1989). Learning skills and the acquisition of knowledge. In A. Lesgold et R. Glaser (éditeurs), Foundations for a psychology of education (pp. 199249). Hillsdale, NJ: Lawrence Erlbaum Associate, Publishers.

19. Boizumault, M. \& Cogérino, G. (2012). La mise en scène corporelle de l'enseignant d'EPS : les communications non verbales au service de l'efficacité de l'enseignant. Revue STAPS, 4(98), 67-79.

20. Boudard, J.-M. (2011). Décrire des pratiques de régulation didactique en éducation physique et sportive : la place des savoirs techniques. eJRIEPS, 24, 53-76.

21. Bouthier, D. (1986). Comparaison expérimentale des effets de différents modèles didactiques des sports collectifs. Dans E.P.S contenus et didactique (pp. 85-90). Paris : SNEP. 
22. Brousseau, G. (1986). Foundations and Methods of the Didactics of the Mathematics. Researches in Didactics of the Mathematics, 7, 33115.

23. Brousseau, G. (1996). Les stratégies de l'enseignant et les phénomènes typiques de l'activité didactique. In R. Noirfalise et M.-J. PerrinGIonan (Éds), Actes de la VIIIe École d'Été de didactique des mathématiques (pp.16-30). Clermont-Ferrand : IREM.

24. Buznic-Bourgeacq, P. (2005). L'expérience de l'enseignant et ses implications didactiques: une étude de cas en EPS. Actes du 5e Colloque international Recherche et formation IUFM de Nantes, France.

25. Buznic-Bourgeacq, P., Terrisse, A., \& Margnes, E. (2007). L'expérience personnelle du professeur d'EPS, approche clinique et implications didactiques : une étude de cas d'une enseignante débutante. eJRIEPS, 11, 29-38.

26. Buznic-Bourgeacq, P., Terrisse, A., \& Lestel, G. (2008). Le poids de l'expérience professionnelle en didactique clinique de l'EPS : analyse comparée de deux enseignantes contrastées. In M.-F., Carnus, C. Garcia-Debanc \& A. Terrisse (dir.), Analyse des pratiques des enseignants débutants : approche didactique (pp.193-213). Grenoble : La pensée sauvage.

27. Buznic-Bourgeacq, P. (2009). La transmission du savoir expérientiel, étude de cas et analyse comparatives en didactique clinique de l'EPS. Thèse de doctorat en Sciences de l'Education, Université Toulouse 3.

28. Carminatti, N. \& Carnus, M.-F. (2019). Le déjà-là proxémique, un analyseur de l'épreuve interactive dans les activités physiques sportives et artistique Varia (p. 5).

29. Carnus, M.-F. (2001). Analyse didactique de processus décisionnel de l'enseignant d'EPS en gymnastique. Une étude croisée. Thèse de doctorat en sciences de l'Éducation non publiée. Université Paul Sabatier, Toulouse III, France.

30. Carnus, M.-F. (2002). Croyances, conceptions, intentions et pratiques usuelles dans l'enseignement de la gymnastique : Le cas de l'ATR et du repérage. In J.-F. Robin et A. Durny (Dir), Travaux d'actualité en activités gymniques et acrobatiques (pp.160-164). Dossiers EPS, 57. Paris : Revue EPS.

31. Carnus, M.-F. (2003). Analyse didactique du processus décisionnel de l'enseignant d'EPS en gymnastique : une étude de cas croisés. In C. Amade-Escot (Éd), Didactique de l'éducation physique, état des recherches (pp. 195-224). Paris : Revue EPS.

32. Carnus, M.-F. (2008). Analyse didactique clinique de pratiques d'enseignants expérimentés et débutants. In M.-F. Carnus, C. Garcia- 
Debanc \& A. Terrisse. (Coord.), Analyse des pratiques des enseignants débutants. Approches didactiques (pp. 213-232). Grenoble : La pensée sauvage,

33. Carnus, M.-F. (2009). Pour une didactique clinique de l'EPS. Perspectives pour la formation des enseignants. Note de synthèse pour l'Habilitation à diriger des recherches. Université Paris Ouest Nanterre le Défense.

34. Carnus, M.-F. \& Terrisse, A. (2013). Didactique clinique de l'EPS. Le sujet enseignant en question. Paris : Editions EPS.

35. Carnus, M.-F. (2015). Le rapport au savoir du sujet enseignant en didactique clinique de l'EPS : un déjà-là décisionnel. In V. Vincent \& M.-F. Carnus (dir.), Le rapport au(x) savoir(s) au cour de l'enseignement. Enjeux, richesse et pluralité (pp.61-73). Bruxelles : De Boeck Supérieur.

36. Cauvin, F. (2002). Repérage des actes de médiation de l'enseignant d'EPS en tennis de table. Impulsion, 3, 63-92.

37. Chevallard, Y. (1985). La Transposition didactique : du savoir savant au savoir enseigné. Revue française de pédagogie , 76 (1), 89-91.

38. Chevallard, Y. (1991). La transposition didactique, du savoir savant au savoir enseigné, avec un exemple d'analyse de la transposition didactique. Grenoble : La Pensée sauvage.

39. Chevallard, Y. (1999). L'analyse des pratiques enseignantes en théorie anthropologique $\mathrm{du}$ didactique. Recherche en didactique des mathématiques, 19(2), 221-266.

40. Clément, R., \& Noels, K. (2006). La communication sociale : Aspects interpersonnels et intergroupes. Les fondements de la psychologie sociale. Québec : Gaëtan Morin Editeur.

41. Defrance, J. (2006). Sociologie du sport ( $5^{\mathrm{e}}$ édition). Paris : La Découverte.

42. Delanoë, M.-H. \& Labridy, F. (1983). Formation des enseignants d'EPS et psychanalyse. Revue EPS, 184, 31-34.

43. Deschryver, N. \& Lameul, G. (2016). Vers une opérationnalisation de la notion de posture professionnelle en pédagogie universitaire. Revue interactionnelle de pédagogie de l'enseignement supérieur, 32, 32-3.

44. Durand, M. (1996). L'enseignement en milieu scolaire. Paris : PUF.

45. Durand, M. (2001). Chronomètre et survêtement. Reflets de l'expérience quotidienne d'enseignants en éducation physique. Paris : Revue EPS.

46. Forest, D. (2006). Analyse proxémique d'interactions didactiques. Carrefours de l'éducation, 1,73-94.

47. Hall, E. T. (1966-1971). La dimension cachée. Double day. 
48. Joas, H. (2001). La créativité de l'agir. In J.M. Baudoin \& J. Friedrich. (Eds). Théories de l'action et éducation. Bruxelles : De Boeck.

49. Jorro, A. (2004). Le corps parlant de l'enseignant. Entente, malentendus, négociations. Symposium : La réflexivité des langages, instruments de travail du professeur et des élèves : points communs et spécificité disciplinaires. AIRDF, Québec (CD-ROM).

50. Jorro, A. (2005). L'éthique de l'évaluateur. Conférence ESEN, Poitiers.

51. Jorro, A. (2006). L'agir professionnel de l'enseignant. In Séminaire de recherche du centre de Recherche sur la formation -CNAM.

52. Joshua, S. (1996). Le concept de transposition didactique n'est-il propre qu'aux mathématiques ? In C. Raisky \& M. Caillot. Au-delà des didactiques, le didactique, Débats autour de concepts fédérateurs (pp. 61-73). Bruxelles : De Boeck.

53. Jourdan, I. (2005). L'évolution de rapport au savoir comme révélateur de la logique de professionnalisation : six études de cas en formation initiale en EPS à l'IUFM Midi Pyrénées. Thèse de doctorat. Université Paul Sabatier. Toulouse 3.

54. Jourdan, I. (2006). Rapport au corps, rapport aux activités physiques, sportives et artistiques et logique professionnelle : deux études de cas en formation initiale en EPS. Aster, 42, 57-78.

55. Kember, D. (1997). A reconceptualisation of the research into university academics conceptions of teaching. Learning and Instruction, 7(3), 255-275.

56. Kohler, M. (2002). Les démonstrations partielles : une technique corporelle pour une meilleure perception du geste. Thèse de doctorat. Université Bordeaux 2.

57. Kugel, P. (1993). How professors develop as teachers. Studies in Higher Education, 18(3), 313-328.

58. Lacan, J. (1966-1967). Le Séminaire, La logique du fantasme. Livre XIV, inédit.

59. Lacan, J. (1968-2001). La méprise du sujet supposé savoir. In Autre écrit. Paris : Seuil.

60. Lacan, J. (1977). The Four Fondamental Concepts of Psycho-Analysis. London: Hogarth Press.

61. Lafont, L. (1994). Modalités sociales d'acquisition d'habiletés motrices complexes. Rôles la démonstration explicitée et d'autres procédures de guidage selon la nature de l'habileté. Thèse de doctorat en psychologie. Paris V.

62. Lameul, G. (2008). Les effets de l'usage des technologies d'information et de communication en formation d'enseignants sur la construction des postures professionnelles. Savoirs, 17, 71-94. 
63. Laot, F. (2009). Formateurs d'adultes et diffusion de la notion du rapport au savoir. Approche socio-historique. Raison éducation, 13.

64. Léal, Y. (2006). L'analyse didactique clinique de pratiques ostensives : une étude de cas croisés en EPS au collège et à l'école primaire. Mémoire de Master non publié. Université Toulouse-Jean Jaurès, Toulouse.

65. Léal, Y. (2012). L'education à la santé dans un enseignement polyvalent. Une étude de cas contrastés en didactique clinique à l'école élémentaire. Thèse de doctorat non publiée. Université de Toulouse 2 Le Mirail.

66. Loizon, D., Margnes, E. \& Terrisse, A. (2005). La transmission des savoirs : le savoir personnel des enseignants. Revue Savoirs, 8, 107123.

67. Loizon, D. (2009). Les filtres personnels dans 1'action didactique. In A. Terrisse \& M.-F. Carnus (dir.). Didactique clinique de l'éducation physique et sportive (EPS). Quels enjeux de savoirs ? (pp. 83-100). Bruxelles : Éditions De Boeck.

68. Loizon, D. (2013). L'enseignant d'EPS et ses filtres déjà-là. In M.-F. Carnus \& A. Terrisse (dir.). Didactique clinique de l'EPS. Le sujet enseignant en question (pp.13-21). Paris : Éditions EPS.

69. Loizon, D. (2016). Analyse didactique clinique des pratiques d'enseignement et de formation. Les effets $d u$ déjà-là expérientiel sportif. Note de synthèse pour l'habilitation à diriger les recherches. Université Toulouse Jean Jaurès, document non publié.

70. Magali, B. \& Geneviève, C. (2012). La mise en scène corporelle de l'enseignant d'EPS. Revue STAPS, 98, 67-79.

71. Marsenach, J. \& Mérand, R. (1987). L'évaluation formative en EPS dans les collèges. Paris : INRP. Rapport scientifique, 2.

72. Maulini, I. \& Olivier. (1999). Le corps à l'école : élément neutre des apprentissages ? Texte publié dans l'Educateur, 8, 32-37.

73. Mehrabian, A. (1971). Silent Messages. Belmont, CA, Wadsworth.

74. Miller J.-A. (2011). L'orientation lacanienne. L'Être et l'Un, enseignement prononcé dans le cadre du département de psychanalyse de l'université Paris VIII. Inédit.

75. Mottier-Lapez, L. (2013). Réguler son enseignement. Pourquoi ? Comment? Colloque IFRES. Liège.

76. Ottet, F. (2016). Analyse de l'engagement corporel de trois enseignants d'EPS en formation initiale : vers la définition d'une technicité corporelle. Communication présentée à Colloque SSRE, Lausanne, Suisse.

77. Passeron, J.-C. \& Revel, J. (2005). Penser par cas, «Enquête». Paris : Ecole des Hautes Etudes en Sciences Sociales. 
78. Perrenoud, P. (1994). La formation des enseignants entre théorie et pratique. Paris : L'Harmattan.

79. Pujade-Renaud, C. (1984). Le corps de l'enseignant dans la classe. 2e édition. Paris : ESF.

80. Quivy, R \& Van Campenhoudt, L. (2006). Manuel de recherche en science sociale. Dunod : Paris.

81. Rey, B., Caffieaux, C., Compère, D., Lammé, A., Persenaire, E., Philippe, J. \& Wallenborn, G. (2003) Etude épistémologique, didactique et textuelle des savoirs enseignés à l'Université et dans les Hautes Ecoles. Rapport de recherche subventionnée. Bruxelles : Ministère de la Communauté française de Belgique, Ministère de l'Enseignement supérieur. (Texte accessible en pdf sur le site de l'Administration générale de l'Enseignement et de la recherche scientifique :

http://www.enseignement.be/prof/dossiers/recheduc/rech1.asp?page= 2).

82. Richmond, V. P., \& McCroskey, J. C. (2004). Nonverbal Behavior in Interpersonal Relations. 5th edition. Boston, MA : Allyn \& Bacon.

83. Robert, M. (2012). L'ostension non verbale en EPS : Analyse didactique clinique des pratiques gestuelles de deux enseignants aux profils contrastés lors de l'enseignement de la rotation avant. Mémoire de Mastère, Université Toulouse Jean Jaurès.

84. Romainville, M. (1998). L’enseignement universitaire a les étudiants qu'il mérite. In M. Frenay, B. Noel, P. Parmentier \& M. Romainville (Ed.). L'étudiant apprenant: grille de lecture pour l'enseignant universitaire (pp. 95-108). Bruxelles : De Boeck.

85. Romainville, M. (2002). L'évaluation des acquis des étudiants dans l'enseignement universitaire. Paris : Rapport établi à la demande du Haut Conseil de l'évaluation de l'école.

86. Robert, M., \& Carnus, M. F. (2013). Les formes ostensives non verbales en gymnastique constructives de la signature professionnelle de deux enseignants d'EPS. In M.F. Carnus, \& A. Terrisse (Eds.), Didactique clinique de l'EPS. Le sujet enseignant en question (pp. 8192). Paris : Revue EPS.

87. Salin, M.-H. (2002). Les pratiques ostensives d'un enseignant des mathématiques comme objet d'analyse du travail du professeur. In $\mathrm{O}$. Venturini, C. Amade-Escot et A. Terrisse (éds.), Etudes des pratiques effectives : l'approche des didactiques (pp. 71-81). Grenoble: La pensée sauvage.

88. Sauret, J.M. (2000). Freud et l'inconscient. Les éditions Milan. 
89. Savoie-Zacq, L. (1996). Triangulation. In A. Muchielli. Dictionnaire des méthodes qualitatives en sciences humaines et sociales (pp. 261262). Paris: Armand Colin.

90. Sensevy, G. (2007). Des catégories pour décrire et comprendre l'action didactique. In G. Sensevy \& Mercier, A. (dir.). Agir ensemble. L'action didactique conjointe du professeur et des élèves (pp. 13-49). Rennes : Presses Universitaires de Rennes.

91. Sghaier, D., Ben Jomaa, H., Mami, M. \& Bouassida, A. (2017). Les pratiques ostensives des enseignants d'EPS révélatrices du rapport au savoir en natation. IOSR Journal of Research \& Method in Education, 7 (4), 36- 44.

92. Sghaier, D., Ben Jomaa, H, Mami, M, Georges Kpazaï \& Bouassida, A. (2019). L'effet des pratiques ostensives sur le rapport au savoir dans l'enseignement de la natation : deux études de cas. eJRIEPS, Numéro spécial, 2, 80-97.

93. Silverman, D. (2009). Doing qualitative research. (3rd Ed.). London: Thousand Oaks; New Delhi: Sage.

94. Simon, F. \& Schmehl-Postaï, A. (2010). Les fonctions des savoirs de l'enseignant : l'exemple d'une situation d'enseignement-apprentissage de l'écriture en CP-CE1. Repères, consulté en ligne le 17 janvier 2020. URL : $\quad$ http://journals.openedition.org/reperes/267 ; DOI : 10.4000/reperes. 267.

95. Terrisse, A (2001). La référence dans l'enseignement de l'éducation physique et sportive. In A. Terrisse (dir.). Didactique des disciplines. Les références au savoir (pp. 119-139). Bruxelles: De Boeck Université.

96. Terrisse, A., Carnus, M.-F. \& Sauvergrain, J.-P. (2002). Le rapport au savoir: point de vue psychanalytique et recherche en didactique de L'EPS. Didactique et rapports aux savoirs (pp.123-130) Actes des

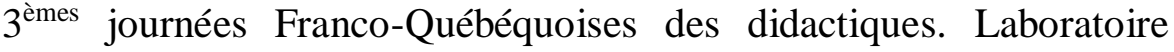
Education et Apprentissage (EDA) Paris : Sorbonne.

97. Terrisse, A. (2003). Prise en compte du sujet et recherches en didactique des sports de combat : bilan et perspectives. Sciences et motricité, 3 (50), 55-79.

98. Terrisse, A. (2009). La didactique clinique en EPS. Origine, cadre théorique et recherches empiriques. In : Terrisse A. \& Carnus M.-F. (Dir.). Didactique clinique de l'éducation physique et sportive (EPS). Quels enjeux de savoir? (pp.13-31). Bruxelles : De Boeck.

99. Terrisse, A. \& Carnus, M.-F. (2009). Didactique clinique de l'éducation physique et sportive. Quels enjeux de savoirs? Paris : De Boeck. 
100. Thomas, C. E., Richmond, V. P., \& McCroskey, J. C. (1994). The Association between Immediacy and Socio-Communicative Style. Communication Research Reports, 11, 107-114.

101. Touboul, A. ; Carnus, M.-F. \& Terrisse, A. (2012). L'influence de l'expérience et de l'expertise sur l'enseignement de l'éducation physique et sportive. Une étude de cas contrastés en savate boxe française. eJRIEPS, 26, 67-93.

102. Touboul, A. (2013). L'impossible à supporter chez une enseignante novice en EPS. In Carnus, M.-F. \& Terrisse (Dir.), A. Didactique clinique de l'EPS : le sujet enseignant en question (pp. 4151). Paris : Editions Revue EPS.

103. Van der Maren, J.-M. (1996). Méthodes de recherche pour l'éducation (2e éd.). Montréal : Les Presses de l'Université de Montréal.

104. Vial, M. (2001). Evaluation et régulation. In G. Figari, M. Achouche \& V. Barthélémy (Dir.). L'activité évaluative: nouvelles problématiques, nouvelles pratiques (pp. 68-78). Bruxelles: De Boeck.

105. Vigarello, G. (1988). Une histoire culturelle du sport. Techniques d'hier et d'aujourd'hui. Paris : Robert Laffont.

106. Vinatier, I. \& Altet, M. (2008). Analyser et comprendre la pratique enseignante. Rennes : Presses universitaires de Rennes.

107. Wittgenstein L. (1994). Remarques sur la philosophie de la psychologie (II). Traduit de l'allemand par G. Granel. Mauvezin : Trans-Europ-Repress, Coll. T.E.R. bilingue, 142. 